Progress In Electromagnetics Research Symposium - Spring

(PIERS), St Petersburg, Russia, 22-25 May. 2017

Pages 2779-2786

http://dx.doi.org/10.1109/PIERS.2017.8262226

http://archimer.ifremer.fr/doc/00437/54829/

(c) 2017 IEEE

\title{
Normalized Radar Backscattering Cross-section and Doppler Shifts of the Sea Surface in Ka-band
}

\author{
Yurovsky Yu. Yu. ${ }^{1}$, Kudryavtsev V. N. ${ }^{1,2}$, Grodsky S. A. ${ }^{3}$, Chapron Bertrand ${ }^{4}$ \\ ${ }^{1}$ FSBSI Marine Hydrophys Inst RAS, Sevastopol, Russia. \\ ${ }^{2}$ Russian State Hydrometeorol Univ, St Petersburg, Russia. \\ ${ }^{3}$ Univ Maryland, Dept Atmospher \& Ocean Sci, College Pk, MD 20742 USA. \\ ${ }^{4}$ IFREMER, Issy Les Moulineaux, France.
}

\begin{abstract}
:
Field data taken from the Black Sea Research platform during 2009-2015 are used to develop a dualco-polarized (VV and $\mathrm{HH}$ ) empirical model for the sea surface normalized radar cross-section (NRCS) and Doppler Velocity (DV) in the Ka-band. A fitting function for the NRCS is the standard truncated Fourier series in azimuth with polynomial coefficients dependent on incidence angle and wind speed. The data are corrected for the angular antenna pattern, which is measured in special calibration procedures. The resulting NRCS model is consistent with other rare Ka-band measurements and agrees well with Ku-band geophysical model functions, in contrast with previously proposed Ka-band models. Surface is subtracted from measured DV using either propeller current meter measurements or surface video recording. The resulting DV model consists of constant and wave-induced (WIDV) parts. The constant part is evaluated based on the time mean of Instantaneous DV (IDV), which is close to the Bragg wave phase speed. The WIDV part is analyzed in terms of Modulation Transfer Function (MTF) for which a separate empirical model is proposed.
\end{abstract}




\section{INTRODUCTION}

Microwave scatterometers have already become a traditional tool for retrieval of near surface winds over the global ocean. A new challenge is the estimation of surface currents that can be extracted from the Doppler frequency shift of radar signal backscattered from the sea surface [1].

Decorrelation time of backscattered signal decreases with shortening of radar wave-length. Therefore besides better spatial resolution, the use of higher frequency radar bands, like the Kaband, allows for higher pulse repetition frequency and better Doppler anomaly separation [2]. In comparison with traditional $\mathrm{C} / \mathrm{X} / \mathrm{Ku}$-bands, the Ka-band scatterometer may improve velocity retrieval accuracy by upto a factor of three [3].

However, the properties of Ka-band backscattering from the sea surface remain poorly investigated. The Normalized Radar Cross-Section (NRCS) is usually described by a Geophysical Model Function (GMF) relating the mean NRCS with wind speed and look geometry. The early GMF [4] for the Ka-band sea surface NRCS was found to be inconsistent with more recent field measurements $[5,6]$, but a better alternative has not been proposed yet.

Doppler features of sea surface radar echo have been evaluated in several studies [7-9], but mainly in order to investigate the Modulation Transfer Function (MTF) of the NRCS. Utilization of the Doppler information for the ocean parameter retrieval demands a model for the mean Doppler velocity (DV), which now is only available for the C-band [10].

Based on data collected during multi-year field experiments from the Black Sea research platform we propose an empirical model for Ka-band NRCS and DV. Although these experiments span multiple years and targeted different physical phenomena, a consistent reanalysis of the entire data set allows to cover a wide variety of geophysical and observation conditions and build an empirical model for various look geometry and wind conditions. Model fitting functions are constructed as a polynomial fit to the coefficients of Fourier series in radar-to-wind direction. The DV part of the model consists of constant and wave-induced parts that are evaluated separately.

\section{EXPERIMENTAL DATA}

The measurements were taken from the Black sea research platform during specialized experiments spanning 2009-2015. Ka-band $(37.5 \mathrm{GHz})$ continuous-wave Doppler radar was used for backscattering measurements. It operated in a hybrid polarization mode with slant $\left(45^{\circ}\right)$ polarization transmitting mode and two receiving channels for vertical (VV) and horizontal (HH) polarization 
components. This configuration implies that cross- polarization may contaminate the received signal. However, this contamination is weak for our conditions and is disregarded (see discussion in [11]).

The raw radar in-phase and quadrature signals at VV and $\mathrm{HH}$ polarization were digitized at $40 \mathrm{kHz}$ rate. The instantaneous NRCS and Doppler shift were computed as zeroth order moment and normalized first order moment of instantaneous Doppler spectra (computed from $0.2 \mathrm{~s}$ long consecutive intervals), respectively.

Supplemental meteorological measurements were made using the Davis Vantage Pro station with air sensors installed at $21 \mathrm{~m}$ height. Neutral $10 \mathrm{~m}$ wind was calculated using the COARE3.0 algorithm [12].

Surface wave properties were recorded by six wire resistance wave gauge antenna. Directional frequency spectra were estimated over 30 min intervals by the maximum likelihood method using the DIWASP package [13]. For the current analysis, we selected only unidirectional wave cases with all waves propagating in the wave peak direction, and excluded cases with significantly different wind and dominant wave directions.

Surface currents were estimated either from video camera co-aligned with the radar or from in situ currents at some reference depth (normally $10 \mathrm{~m}$ ). Video measurements are used as a primary source for the surface currents, which is estimated as the translation velocity of long-living surface markers (small standalone bubbles, teared foam spots, small debris, etc). In the absence of video records, we rely on in situ $10 \mathrm{~m}$ current meter data corrected for an empirical estimate of wind-induced shear in the upper $10 \mathrm{~m}$ column $(\approx 2.3 \%$ of wind speed $)$.

Continuous radar records were subdivided into 5 min samples. The total number of samples is about 1500 for all incidence angels ranging from $0^{\circ}$ to $70^{\circ}$. After elimination of samples with unknown wave conditions, the total number of remaining samples is about 500. Almost $35 \%$ of them were accompanied either by surface video records or current meter measurements.

\section{NORMALIZED RADAR CROSS-SECTION}

Our radar has a specific angular antenna pattern flattened in the vertical/horizontal direction at $\mathrm{HH} / \mathrm{VV}$ polarization, respectively. Because the sea surface NRCS has a rapid incidence angle dependence, the radar antenna pattern leads to artificial changes in measured NRCS. To correct for the antenna pattern, we fit the data by solving a non-linear equation:

$$
\sigma_{\text {eff }}(\theta, \phi, U)=\frac{\int \Gamma_{\text {eff }}(x, y) \sigma^{\circ}(x, y, U) \mathrm{d} x \mathrm{~d} y}{\int \Gamma_{\text {eff }}(x, y) \mathrm{d} x \mathrm{~d} y},
$$

where $\Gamma_{\text {eff }}$ is the radar pattern projected on the ground plane, $\sigma^{\circ}$ is the "true" sea surface NRCS, and $\sigma_{\mathrm{eff}}$ is the measured NRCS, $\theta$ is the incidence angle, $U$ is the wind speed.

The true NRCS is expanded into truncated Fourier series [14,15] of azimuth:

$$
\sigma^{\circ}=A_{0}(\theta, U)+A_{1}(\theta, U) \cos \phi+A_{2}(\theta, U) \cos 2 \phi,
$$

where the coefficients $A_{j}$ are 4 th degree polynomial functions of incidence angle and linearly depend on logarithm of wind speed. The first guess for the iterative solution of (1) is the fit (2) to the measured, rather than true, NRCS ( $\Gamma_{\text {eff }}$ is supposed to be delta-function in (1)).

Because of the lack of measurements at small incidence angles $\left(\theta<20^{\circ}\right)$ the resulting model fails to reproduce the correct polarization ratio in this angle range. At high $\theta$, the maximal effective incidence angle is decreased due to the integration over the angular pattern. Thus the fit is reliable for $25^{\circ}<\theta<65^{\circ}$ and $3<U<18 \mathrm{~m} / \mathrm{s}$, both defined by the radar sensitivity and observed conditions.

The resulting empirical fit, refered to as a Ka-band Dual co-Polarized Model (KaDPM), is compared to the only available Ka-band GMF [4] and adjacent Ku-band GMFs [16], [17] (Fig. 1). The onmi-directional NRCS, $A_{0}$, is quite close in the Ka- and $\mathrm{Ku}$-bands for all models except for [4], which demonstrate systematically lower, by $5-6 \mathrm{~dB}$, values (Fig. 1a,d).

The ratio, $A_{1} / A_{0}$, which is responsible for upwind-downwind asymmetry, is different in the Ka- and Ku-bands. While Ku-band $A_{1} / A_{0}$ at high incidence angles $\left(\theta>40^{\circ}\right)$ never exceeds 0.2 and 0.4 at $\mathrm{VV}$ and $\mathrm{HH}$ polarizations, respectively, in the Ka-band asymmetry is almost twice as strong. At lower incidence angles $\left(\theta<40^{\circ}\right)$ the $A_{1}$ becomes negative indicating that the NRCS is higher in downwind direction. Such negative $A_{1}$ may suggest enhanced backscattering from rear wave slopes. The upwind-crosswind asymmetry, $A_{2} / A_{0}$, is roughly consistent in both bands at 

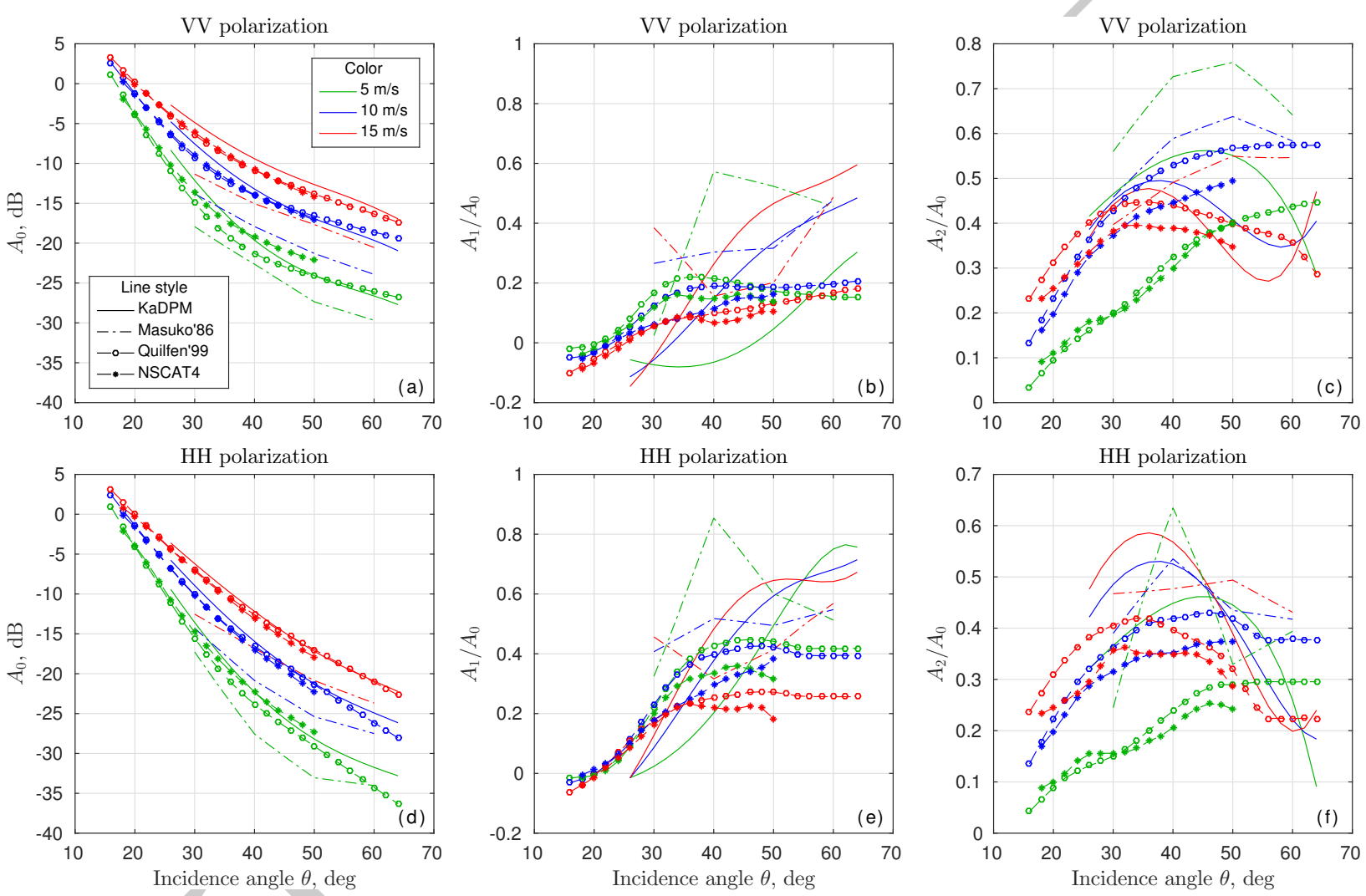

Figure 1: NRCS models overview: (a,d) omni-directional NRCS, $A_{0}$, (b,e) upwind-downwind asymmetry, $A_{1} / A_{0}$, (c,f) upwind-crosswind asymmetry, $A_{2} / A_{0}$, versus incidence angle for wind speed $5 \mathrm{~m} / \mathrm{s}$ (green), 10 $\mathrm{m} / \mathrm{s}$ (blue), and $15 \mathrm{~m} / \mathrm{s}$ (red) at VV (top row) and $\mathrm{HH}$ polarization (bottom row).

rather strong winds $U>10 \mathrm{~m} / \mathrm{s}$, while at $U=5 \mathrm{~m} / \mathrm{s}$ Ka-band demonstrates the more anisotropic behavior.

Following [18], the dual co-polarization radar data allow to partition the NRCS into polarized (Bragg backscattering) and non-polarized (specular reflection from the regular surface and breaking waves) components. Such partitioning conducted for the KaDPM and Ku-band GMFs [11] shows that relative contribution of the Bragg-like term is higher in the Ka-band than in the Ku-band. This effect is attributed to an increased small-scale roughness produced by the parasitic capillaries (bound ripple) specific to Ka-band Bragg wave-number range.

\section{DOPPLER VELOCITY}

The sea surface can be represented as an ensemble of non-coherent scatterers with their own NRCS and line-of-sight velocity (LOSV). Each of the two is represented as a sum of mean and fluctuating components. The mean Weighted DV (WDV) measured by a radar with an infinitely large illumination swath is

$$
\mathrm{WDV}=\bar{v}+\frac{\overline{v^{\prime} \sigma^{\prime}}}{\bar{\sigma}},
$$

where $\bar{v}$ and $\bar{\sigma}$ are the mean background LOSV and NRCS, $v^{\prime}$ and $\sigma^{\prime}$ are the fluctuations of local LOSV and NRCS. The second term in (3), a wave-induced DV (WIDV), is responsible for the impact of correlation between $\sigma^{\prime}$ and $v^{\prime}$, due to the geometrical tilting, aero- and hydrodynamic modulation of scattering roughness.

For an infinitely narrow radar pattern, the correlator in (3) approaches zero, and a simple arithmetic mean of Instantaneous DV (IDV), yields the mean scatterer LOSV, IDV $=\bar{v}$. Nevertheless, our particular radar has the finite radar pattern width resulting in spatial averaging of waves shorter 
than footprint size:

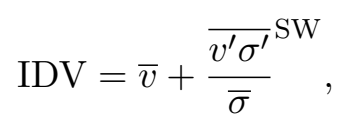

where $\overline{(\ldots)}$ SW denotes averaging over short waves (SW) with a wave-length below the footprint size.

The mean scatterer LOSV can be expressed as a sum of background surface drift current LOSV, $v_{\mathrm{dr}}=V_{\mathrm{dr}} \cos \left(\phi_{\mathrm{dr}}\right)$, and the inherent scatterer velocity, $v_{\mathrm{sc}}$, projected on the radar view direction

$$
\bar{v}=\left(v_{\mathrm{dr}}+v_{\mathrm{sc}}\right) \sin \theta
$$

where $V_{\mathrm{dr}}$ is the surface current, $\phi_{\mathrm{dr}}$ is the radar-to-current azimuth.

The inherent scatterer velocity $v_{\text {sc }}$ depends on the backscattering mechanism. At low incidence angles, when the backscattering is mainly supported by quasi-specular reflections, the scatterer velocity is the mean specular point $\operatorname{LOSV} v_{\mathrm{sc}}=c_{\mathrm{sp}}[19,20]$. At moderate $\theta$ the backscattering is resonant, thus the scatterers are Bragg wave trains riding on longer waves. Their inherent velocity is Bragg wave phase velocity, $v_{\mathrm{sc}}=c_{\mathrm{br}}= \pm\left(g / k_{\mathrm{br}}+\gamma k_{\mathrm{br}}\right)^{1 / 2}$, where $g$ is the gravity acceleration, $\gamma$ is the surface tension coefficient, $k_{\mathrm{br}}=2 k_{\mathrm{r}} \sin \theta$ is the Bragg wave-number. At high $\theta$ the backscattering from breaking waves becomes important, thus the scatterer velocity is also affected by mean wave breaking LOSV, $v_{\mathrm{sc}}=c_{\mathrm{wb}}$.

The overview of measured IDV and WDV is given in Fig. 2 for drift-corrected (solid symbols) and non-corrected (transparent symbols) estimates. The IDV and WDV are generally defined by observation geometry (incidence angle and azimuth) and to a lesser extent by sea state. The WDVIDV difference is caused by WIDV contribution, which is higher at small $\theta$ as can be anticipated from general $\sigma(\theta)$ dependence. This is confirmed by a higher WDV clearly seen at $20^{\circ}<\theta<30^{\circ}$. In fact this leads to almost constant $|\mathrm{WDV}| \approx 0.4 \mathrm{~m} / \mathrm{s}$ at $\mathrm{VV}$ polarization for $\theta>20^{\circ}$ in both upwind and downwind directions. In contrast, HH WDV increases by a factor of two at high $\theta=70^{\circ}$ in the upwind direction, while still it is almost constant in downwind direction. This can be explained by the azimuthally anisotropic impact of wave breaking that has larger inherent speed, $c_{\mathrm{wb}}>c_{\mathrm{br}}$, and is stronger in the upwind direction $\left(\sigma_{\mathrm{wb}}\left(\phi=0^{\circ}\right) \gg \sigma_{\mathrm{wb}}\left(\phi=180^{\circ}\right)\right)$.

\subsection{Constant Doppler Velocity}

To access the constant part of DV, $\bar{v}$ in $(3,4)$, we notice that IDV is quite close to Bragg model prediction (Fig. 2a,b). Thus it can be represented as a sum of surface currents, Bragg wave phase speed, and a residual term caused by: i) the WIDV arising from SW averaging within the footprint, ii) non-Bragg scatterers that have LOSV different from Bragg wave phase speed (specular points at small $\theta$ and wave breaking at high $\theta$ ). We adopt the following model for the IDV

$$
\mathrm{IDV}=v_{\mathrm{dr}}+c_{\mathrm{br}} \sin \theta+\Delta c
$$

The $\Delta c$ term is fitted by the polynomial empirical function of incidence angle and azimuth (wind speed dependence is omitted). The residual DV, $\Delta c$, is generally positive in the upwind direction and negative in the downwind direction (Fig. 2). Its magnitude decreases to zero at large $\theta$, except for $\mathrm{HH}$ polarization upwind case for which the residual term increases towards large $\theta>60^{\circ}$. The latter is explained by fast non-Bragg breaking wave scatterers, which partial contribution to the total NRCS is stronger at $\mathrm{HH}$ polarization.

\subsection{Wave-Induced Doppler Velocity}

The WIDV is determined by the correlation between fluctuating parts of $v_{\mathrm{D}}$ and $\sigma$. Both $v_{\mathrm{D}}^{\prime}$ and $\sigma^{\prime}$ are associated with the long surface waves $(\mathrm{LW})$ and can be analyzed in terms of Modulation Transfer Function (MTF) [7,21,22], which linearly relates variations of NRCS with LW slopes. The Doppler channel is sensitive to the LW orbital velocity, and thus can act as a wave probe [7]. For deep water waves the MTF is:

$$
M=\frac{\sigma^{\prime}}{\bar{\sigma} a k}=\frac{g G}{\bar{\sigma} \omega} \frac{S_{\sigma v}}{S_{v v}},
$$

where $a, k$, and $\omega$ are the LW amplitude, wave-number, and angular frequency, respectively, $g$ is the gravity acceleration, $G=\cos \phi \sin \theta+i \cos \theta$ is the geometrical coefficient describing the projection of the orbital velocity vector onto the line-of-sight, and $S_{x y}$ is the cross-spectrum of $x$ and $y$. 

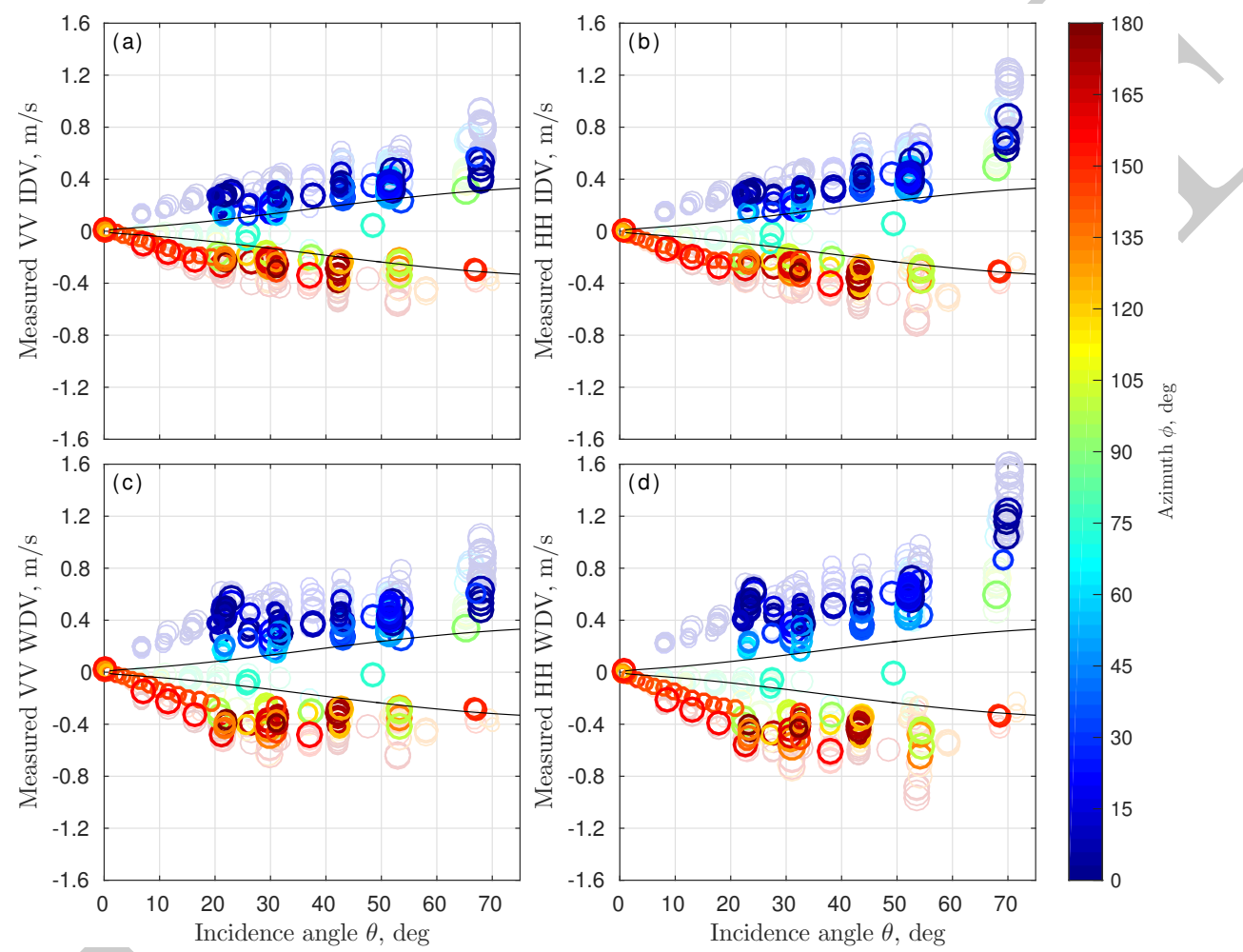

Figure 2: The measured (a,b) IDV and (c,d) WDV versus incidence angle for drift-corrected (solid symbols) and non-corrected (transparent symbols) estimates. Solid lines correspond to the LOSV Bragg wave phase velocity, $c_{\mathrm{br}} \sin \theta$. Symbol size corresponds to the wind speed varying from $3 \mathrm{~m} / \mathrm{s}$ to $18 \mathrm{~m} / \mathrm{s}$.

In these terms, the WIDV reads

$$
\frac{\overline{v^{\prime} \sigma^{\prime}}}{\bar{\sigma}}=\operatorname{Re} \int g^{-1} G^{*} \omega^{3} M S_{z z} \mathrm{~d} \omega .
$$

Observed MTF magnitude and phase are both found to be almost constant within the LW frequency range from wave peak to SW frequency scale. They are fitted by polynomial functions of incidence angle $\theta$, azimuth $\phi$, and logarithm of wind speed (Fig. 3).

In line with previously reported measurements (see e.g. [22]), the magnitude of MTF is generally higher at $\mathrm{HH}$ polarization. It has a minimum in the crosswind direction (Fig. 3a,c), and is $\approx 1-2 \mathrm{~dB}$ higher in downwind/upwind direction at small/high $\theta$. The dependence of MTF magnitude on $\theta$ is complex and probably reflects switching among dominant backscattering regimes. A noticeable jump in MTF magnitude is seen at $\theta>60^{\circ}$ (especially at $\mathrm{HH}$ polarization) that is in line with observed difference between HH WDV and IDV at these incidence angles (Fig. 2).

The MTF phase reflects the angle between local NRCS and wave elevation. Primarily the phase depends on the radar-to-wave azimuth. For the Bragg regime $\left(25^{\circ}<\theta<60^{\circ}\right)$ the NRCS peak locates at $\pm 50^{\circ}$ on the front/rear LW slope for the upwind/downwind direction. This suggests rather strong hydrodynamical modulation that shifts the NRCS peak towards the LW crest from the maximum of LW slope $\left( \pm 90^{\circ}\right)$, which otherwise could be anticipated based on the "pure" tilt modulation mechanism.

\subsection{Empirical model}

In order to smooth our uneven data set the constant DV component and the WIDV component are fitted in order to construct an empirical model for the total WDV. Our simplified approach partitions the total WDV into four parts including: i) surface current; ii) Bragg wave phase velocity, $c_{\mathrm{br}}$; iii) a deviation of from Bragg wave phase velocity due to the SW spatial modulation within the radar footprint, specular reflection contribution at low $\theta$ and breaking wave at high $\theta$; iv) wave induced component, WIDV. Note, that first three components are included in the IDV (6). Model parameters are fitted against incidence angle, azimuth (swell cases are excluded), and wind speed. 



Figure 3: Radar MTF (a,c) magnitude and $(\mathrm{b}, \mathrm{d})$ phase versus incidence angle $\theta$ for different azimtuhs for $(\mathrm{a}, \mathrm{b}) \mathrm{VV}$ and $(\mathrm{c}, \mathrm{d}) \mathrm{HH}$ polarization. Fitting functions are shown by lines for $U=10 \mathrm{~m} / \mathrm{s}$. Symbol size corresponds to the wind speed varying from $3 \mathrm{~m} / \mathrm{s}$ to $18 \mathrm{~m} / \mathrm{s}$.

The WIDV is evaluated using the Toba's spectrum [23], $S_{z z}(f)=0.085 \cdot\left(g / 8 \pi^{3}\right)(U / 28) f^{-} 4$, with the peak frequency corresponding to the median wave age for data set, $c_{\mathrm{p}} / U_{10} \approx 0.7$.

In general, the WDV values obtained using the Toba's spectrum are close to observed values (Fig. 4). Due to the lack of surface current measurements, the data with unknown deep currents are also included in Fig.4. At small incidence angles, the WDV is proportional to $\sin \theta$ because look geometry. The magnitude of WDV is higher at $\mathrm{HH}$ in comparison to VV polarization. At light to moderate winds, $U<10 \mathrm{~m} / \mathrm{s}$, and $30^{\circ}<\theta<60^{\circ}$ the upwind/downwind VV WDV is quite stable with magnitudes varying within $0.4-0.5 \mathrm{~m} / \mathrm{s}$. At higher $\theta>60^{\circ}$ there exists some increase in the WDV magnitude, but it is observed only in upwind direction and is more pronounced at $\mathrm{HH}$ polarization.

An interesting feature of the WDV is its incidence angle dependence in the crosswind direction. It is weakly negative, WDV $>-0.2 \mathrm{~m} / \mathrm{s}$, at $\theta<50-55^{\circ}$, and switches to positive values at higher $\theta$. In the crosswind direction, all WDV components are zero, except for WIDV. This wave-induced component can be negative/positive if the NRCS maximum is shifted onto the rear/front LW slope, respectively. The observed crosswind WDV suggests that NRCS maximum locates on the rear slope at small $\theta$, but moves on the forward slope at high $\theta$.

\section{DISCUSSION AND SUMMARY}

Based on field data, we present a new empirical dual-polarized model for the sea surface NRCS and Doppler shift in the Ka-band. The radar dual co-polarized NRCS measurements provide a unique possibility to access the Bragg wave spectra in the wavenumber range not-resolvable by any other technique. There are no well-established Ka-band NRCS models, except [4] and its modified version [24]. However, a number of Ka-/Ku-band measurements including those at near nadir incidence angles $[6,25]$ suggest a closer correspondence between $\mathrm{Ku}$ - and Ka-band NRCS than that predicted by [4]. In general, the KaDPM model is in better agreement with well-established Ku-band GMFs [16,17]. The separation of the NRCS into polarized and non-polarized terms [18] 

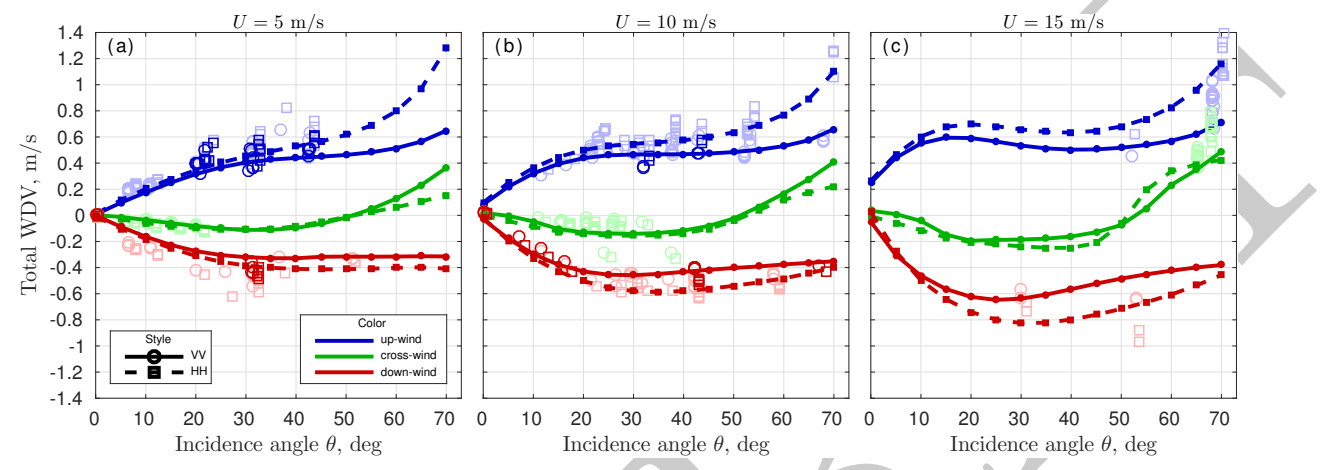

Figure 4: Model predicted WDV for (a) $U=5 \mathrm{~m} / \mathrm{s}$, (b) $U=10 \mathrm{~m} / \mathrm{s}$, and (c) $U=15 \mathrm{~m} / \mathrm{s}$ for upwind (blue), crosswind (green), and downwind (red) direction. Symbols (circles for VV, and squares for HH polarization) are measured WDV corrected for surface currents. Observations are binned $\pm 2^{\circ} \mathrm{m} / \mathrm{s}$ in wind speed and $\pm 15^{\circ}$ in azimuth. Transparent symbols are not-corrected WDV observations.

(performed in [11] for the KaDPM) indicates an anticipated local spectrum peak at wave-numbers about $1000 \mathrm{rad} / \mathrm{m}$ due to parasitic ripple generation.

The mean Doppler velocity (DV) is a key parameter for the estimation of surface currents from satellite-based radars. However, it is still much less investigated than the NRCS. Theoretical and empirical models are available only for the C-band $[10,20]$. This paper provides an empirical model of Ka-band DV based on platform measurements. Besides the background surface currents, the model includes two components that describe the inherent scatterer velocity and the wave-induces Doppler velocity (WIDV). The first term is partitioned into Bragg wave phase velocity and residual term responsible for the deviation of scatterer velocity from Bragg wave phase velocity. At high incidence angles, it is found that DV is higher at $\mathrm{HH}$ polarization and in the upwind direction. It indicates a contribution of anisotropic non-Bragg scatterers associated with breaking waves. The second term, WIDV, is described in terms of the traditional radar MTF that is estimated for various look geometries and wind conditions. The WIDV contribution is expectedly high at high wind (wave) conditions and at small to moderate incidence angles $15^{\circ}<\theta<30^{\circ}$, where the tilt modulation of the NRCS is strong. Potentially this can be used to include Doppler parameters into procedures of wave retrieval from radar data. The MTF itself contains a valuable information on the physics of short wave modulation by longer waves, which can be accessed in future studies.

\section{ACKNOWLEDGMENT}

The work was supported by Russian Science Foundation grant 15-17-20020, and by NASA/Physical Oceanography grant NNX15AG40G.

\section{REFERENCES}

1. Chapron, B., F. Collard, and F. Ardhuin, "Direct measurements of ocean surface velocity from space: Interpretation and validation," J. Geophys. Res. (Oceans), Vol. 110, No. C7, NIL_76-NIL_92, 2005.

2. Boisot, O., L. Amarouche, J. C. Lalaurie, and C. A. Guerin, "Dynamical Properties of Sea Surface Microwave Backscatter at Low-Incidence: Correlation Time and Doppler Shift," IEEE Trans. Geosci. Rem. Sens., Vol. 54, 7385-7395, 2016.

3. Rodriguez, E., D. Perkovic-Martin, C. Baldi, K. Cooper, N. Majurec, M. Neumann, F. Nicaise, and G. Farquharson, "Ka-band doppler scatterometer for measurements of ocean surface vector winds and currents," in Proceedings of Earth Science Technology Forum ESTF2014, Leesburg, Virginia, USA, October 2014.

4. Masuko, H., K. Okamoto, M. Shimada, and S. Niwa, "Measurement of microwave backscattering signatures of the ocean surface using X band and Ka band airborne scatterometers," J. Geophys. Res. (Oceans), Vol. 91, 13065-13084, 1986.

5. Walsh, E. J., D. C. Vandemark, C. A. Friehe, S. P. Burns, D. Khelif, R. N. Swift, and J. F. Scott, "Measuring sea surface mean square slope with a 36-GHz scanning radar altimeter," J. Geophys. Res. (Oceans), Vol. 103, 12587-12601, 1998. 
6. Vandemark, D., B. Chapron, J. Sun, G. H. Crescenti, and H. C. Graber, "Ocean Wave Slope Observations Using Radar Backscatter and Laser Altimeters," J. Phys. Oceanogr., Vol. 34, 2825-2834, 2004.

7. Keller, W. C. and J. W. Wright, "Microwave scattering and the straining of wind-generated waves," Rad. Sci., Vol. 10, 139-147, 1975.

8. Feindt, F., J. Schroter, and W. Alpers, "Measurement of the ocean wave-radar modulation transfer function at $35 \mathrm{GHz}$ from a sea-based platform in the North Sea," J. Geophys. Res. (Oceans), Vol. 91, No. C8, 9701-9708, 1986.

9. Plant, W. J., E. A. Terray, R. A. Petitt, and W. C. Keller, "The dependence of microwave backscatter from the sea on illuminated area: Correlation times and lengths," J. Geophys. Res. (Oceans), Vol. 99, 9705-9723, 1994.

10. Mouche, A. A., F. Collard, B. Chapron, K. F. Dagestad, G. Guitton, J. A. Johannessen, V. Kerbaol, and M. W. Hansen, "On the Use of Doppler Shift for Sea Surface Wind Retrieval From SAR," IEEE Trans. Geosci. Rem. Sens., Vol. 50, 2901-2909, 2012.

11. Yurovsky, Yu. Yu., V. N. Kudryavtsev, S. A. Grodsky, and B. Chapron, "Ka-Band Dual Copolarized Empirical Model for the Sea Surface Radar Cross Section," IEEE Trans. Geosci. Rem. Sens., doi:10.1109/TGRS.2016.2628640, 1-19, 2016.

12. Fairall, C. W., E. F. Bradley, J. E. Hare, A. A. Grachev, and J. B. Edson, "Bulk Parameterization of Air Sea Fluxes: Updates and Verification for the COARE Algorithm," J. Cli., Vol. 16, 571-591, 2003.

13. "Diwasp, a directional wave spectra toolbox for matlab: User manual," Research Report WP-1601-DJ (V1.1), Centre for Water Research, University of Western Australia.

14. Ulaby, F. T., R. K. Moore, and A. K. Fung, Microwave Remote Sensing: Active and Passive., Dedham, USA, Artech House, Vol. 3, 1065-2126, 1986.

15. Wentz, F. J., and D. K. Smith, "A model function for the ocean-normalized radar cross section at 14 GHz derived from NSCAT observations," J. Geophys. Res. (Oceans), Vol. 104, 11499-11514, 1999.

16. Quilfen, Y., B. Chapron, A. Bentamy, J. Gourrion, T. Elfouhaily, and D. Vandemark, "Global ERS 1 and 2 and NSCAT observations: Upwind/crosswind and upwind/downwind measurements," J. Geophys. Res. (Oceans), Vol. 104, 11459-11469, 1999.

17. "NSCAT-4 geophysical model function." Royal Netherlands Meteorological Institute (KNMI), Available online: http://projects.knmi.nl/scatterometer/nscat_gmf/

18. Kudryavtsev, V. N., B. Chapron, A. G. Myasoedov, F. Collard, and J. A. Johannessen, "On Dual CoPolarized SAR Measurements of the Ocean Surface," IEEE Geosci. Rem. Sens. Lett., Vol. 10, 761-765, 2013.

19. Longuet-Higgins, M. S. "The Statistical Analysis of a Random, Moving Surface," Royal Soc. London Phil. Trans. Ser. A, Vol. 249, 321-387, 1957.

20. Hansen, M. W., V. Kudryavtsev, B. Chapron, J. A. Johannessen, F. Collard, K.-F. Dagestad, and A. A. Mouche, "Direct measurements of ocean surface velocity from space: Interpretation and validation," Rem. Sens. Env., Vol. 120, 113-122, 2012.

21. Plant, W. J. "The Modulation Transfer Function: Concept and Applications," Radar Scattering from Modulated Wind Waves, 155-172, 1989.

22. Kudryavtsev, V., D. Hauser, G. Caudal, and B. Chapron, "A semiempirical model of the normalized radar cross section of the sea surface, 2. Radar modulation transfer function," J. Geophys. Res. (Oceans), Vol. 108, No. C08055, doi:10.1029/2001JC001004, 2003.

23. Toba, Y., "Local balance in the air-sea boundary processes," J. Oceanogr. Soc. Japan, Vol. 29, No. 5, 209-220, 1973.

24. Nekrasov, A. and P. Hoogeboom, "A Ka-Band Backscatter Model Function and an Algorithm for Measurement of the Wind Vector Over the Sea Surface," IEEE Geosci. Rem. Sens. Lett., Vol. 2, 23-27, 2005.

25. Tanelli, S., S. L. Durden, and E. Im, "Simultaneous Measurements of Ku- and Ka-Band Sea Surface Cross Sections by an Airborne Radar," IEEE Geosci. Rem. Sens. Lett., Vol. 3, 359-363, 2006. 